\title{
Dislocation structures forming due to an interaction of slip band with an elastic field of wedge disclination
}

\author{
J. V. Svirina ${ }^{\dagger, 1}$, V.N. Perevezentsev ${ }^{1}$, V. V. Rybin ${ }^{2}$ \\ †j.svirina@mail.ru \\ ${ }^{1}$ Mechanical Engineering Research Institute, RAS, 85 Belinskiy St., Nizhny Novgorod, 603024, Russia \\ ${ }^{2}$ Institute of Applied Mathematics and Mechanics, Peter the Great Polytechnic University, 29 Politekhnicheskaya St., \\ St. Petersburg, 195251, Russia
}

\begin{abstract}
Rotational-type mesodefects - strain induced junction disclinations, are formed at joints and ledges of high-angle grain boundaries during plastic deformation of polycrystals. The strength of these mesodefects increases with deformation and, as a result, they create non-uniform elastic stress fields, which significantly affect the processes of structure formation, strain hardening and material's fracture. In the present work, the interaction of a slip band propagating in a grain body under the action of an external stress field with an elastic field of wedge disclination is investigated by the discrete dislocation dynamics simulation method. The simulation results show that the behavior of the front of the band has common regularities. For a given distance between the slip band and the disclination $y_{0}$, the behavior of the dislocation cluster in the front of the band essentially depends on the distance between the slip planes of the dislocations $h$. For small $h$, it is similar to the behavior of a dislocation pile-up. However, the maximum density of dislocations occurs not in the head of the cluster, as in the classical blocked dislocation pile-up, but in its central part. An increase in the distance $h$ between dislocations slip planes leads to a "splitting" of the front of the band. For larger $h$ the front of the band transforms into a broken from both sides dislocation wall. The minimal distance $h_{c}$, above which such dislocation wall is formed, depends on the disclination strength $\omega_{0}$ and the distance $y_{0}$. The maximal misorientation of the wall is equal to the disclination strength $\omega_{0}$ at $y_{0}=0$. With an increase in the external stress, the wall overcomes the force barrier of the disclination and moves as a whole to the lateral surface of the crystal. Multiple reiteration of this process leads to the possible appearance of the system of broken from both sides dislocation walls in the body of grain.
\end{abstract}

Keywords: computer simulation, slip band, disclination.

\section{Introduction}

It is known that during plastic deformation of polycrystalline solids, rotational-type mesodefects - strain induced junction disclinations - are formed at the joints and ledges of high-angle grain boundaries [1-3]. The strength of these mesodefects increases during deformation, as a result of which they create non-utiform elastic stress fields that significantly affect the processes of structure formation, strain hardening, and fracture of materials. A consistent approach to constructing theoretical models of the mechanical behavior of a material under large deformations is to consider the regularities and features of the behavior of dislocations and dislocation ensembles in stress fields of mesodefects that evolve during deformation. Within the framework of this approach, it was shown in [4-7] that near strain induced junction disclinations in the volume of grains mesoscopic regions appear, characterized by a high density of the dislocation charge, in which well-defined broken dislocation boundaries emanating from the joints in the grain body are formed. Existing theoretical models provide good explanation of the reliably established experimental fact that at the initial stages of polycrystal fragmentation (for characteristic values of strain $\varepsilon \geq 0.2$ ), strain induced broken boundaries are formed near joints and ledges of grain boundaries $[1,8,9]$. An analysis of the contribution of junction disclinations to the strain hardening of polycrystals was carried out in a number of theoretical works [10-12]. In [10], the analysis was carried out in the continuum approximation using a system of balance equations for dislocations in the volume of the grain and disclinations at the joints of strain induced fragments. It was shown that the disclination model describes well the fourth stage of strain hardening of polycrystals. In $[11,12]$, the contribution of junction disclinations to the deforming stress was estimated using the method of discrete dislocation dynamics simulations [13].

The conditions for the passage of pile-up of lattice dislocations through the force barrier created by junction disclination were studied, and the dependences of the deforming stress on the disclination strength and the parameters characterizing the accumulation of dislocations were calculated. The conditions for the nucleation of cracks in elastic fields of junction disclinations were considered in [14-18]. The influence of the elastic field of junction disclination on the nucleation of microcracks in the head of a pile-up of lattice dislocations retarded near the grain boundary was considered in [11]. 
In the present work, in the framework of the discrete approach, the interaction of dislocations with the elastic field of a wedge disclination in the practically important case of localization of deformation in the grain body in the form of slip bands, was considered.

\section{Model Description}

Let us consider the interaction of a plastic shear localized in a slip band of width $H$ with a wedge disclination of strength $\omega$ located at the center of the lower boundary of the model grain (Fig. 1). Let the plastic deformation in the band be carried out by the movement of edge dislocations in the field of external stresses $\sigma^{\text {ext }}$, in slip planes spaced apart by the same distance $h$ (Fig. 1). In this case, the plastic shear front can be described as clusters of $N=H / h$ dislocations distributed over the width of the band. Let us analyze the behavior of such a cluster during the passage of the band through the elastic field of the junction disclination using the method of discrete dislocation dynamics simulations [13].

The equation of motion of the $k$ dislocation with the Burgers vector $b$ of the cluster (the coordinate system is shown in Fig. 1) in the slip plane, which is separated from the disclination by the distance $y_{k}=y_{0}+h(k-1), k=1,2, \ldots, N$, in the quasi-viscous approximation has the form [12]:

$$
V_{k}=M b \sigma_{x y}^{\Sigma}\left(x_{k}, y_{k}\right)
$$

Here: $V_{k}$ is the dislocation velocity, $M$ is the dislocation mobility,

$$
\sigma_{x y}^{\Sigma}=\sigma_{x y}^{e x t}+\sigma_{x y}^{\Delta}+\sigma_{x y}^{d i s l}
$$

- the total field acting on the $k$ dislocation of the cluster from the side of homogeneous external stresses $\sigma_{x y}^{e x t}$, internal stresses from the wedge disclination [19]:

$$
\sigma_{x y}^{\Delta}=-D \omega \frac{x_{k} y_{k}}{\left(x_{k}^{2}+y_{k}^{2}\right)}
$$

and from other dislocations of the cluster:

$$
\sigma_{x y}^{d i s l}=D b \sum_{i \neq k} \frac{\left(x_{i}-x_{k}\right)\left(\left(x_{i}-x_{k}\right)^{2}-\left(y_{i}-y_{k}\right)^{2}\right)}{\left(\left(x_{i}-x_{k}\right)^{2}+\left(y_{i}-y_{k}\right)^{2}\right)^{2}},
$$

(where: $1 \leq i \leq N-1), D=G / 2 \pi(1-v), G$ is the shear modulus, $v$ is the Poisson's ratio. As can be seen from Eq. (3), the force acting in the slip plane on the $k$ dislocation from the

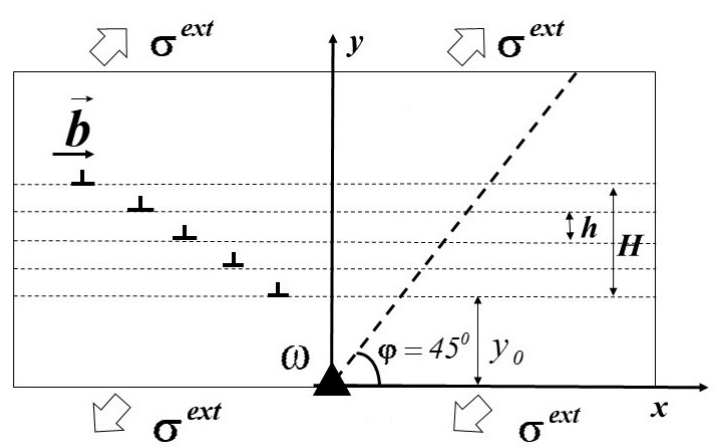

Fig. 1. Schematic plot of the slip geometry of dislocations in the band. The wedge disclination is located in the center of the lower grain boundary. disclination is positive in the crystal region to the left of the disclination $\left(x_{k}<0\right)$ and negative to the right of it $\left(x_{k}>0\right)$ (Fig. 1). Wherein, in the area to the left of the disclination the force $\sigma_{x y}^{e x t} b$ acting on the dislocation from the external stresses coincides in direction with the force acting on it from the disclination, and in the area to the right of disclination these forces are directed opposite to each other. Thus, in the region $x_{k}>0$, the moving dislocation is inhibited by the disclination field.

It is not difficult to see that a steady state of equilibrium of a single dislocation is possible only if the condition $\sigma^{\text {ext }}<\sigma_{c}^{*}$ is satisfied, where $\sigma_{c}^{*}=D \omega / 2$ is the maximum shear stress from disclination achieved on the beam conducted through the disclination at an angle $\varphi=45^{\circ}$ (Fig. 1). Accordingly, the condition of the passage of the plastic shear, realized by the movement of a single dislocation, through the force barrier created by the disclination, has the form: $\sigma^{\text {ext }} \geq \sigma_{c}^{*}$. To take into account dry friction when simulating the motion of a dislocation cluster, it was assumed that there is a threshold stress of dislocation start $\sigma^{\text {th }}$. When $\sigma_{x y}^{\sum} \leq \sigma^{t h}$, the dislocation velocity was equal to zero. The calculations were carried out at $\sigma^{\text {th }}=5 \cdot 10^{-4} \mathrm{D}$. The calculated region of the crystal was selected in the form of a rectangle with dimensions of $10 \times 3 \mu \mathrm{m}$.

The calculation of the equilibrium configuration of the cluster in the case when the magnitude of the external stress field is insufficient for the cluster to overcome the disclination force barrier was carried out by the method of successive iterations in time. The cluster of dislocations was formed by successive generation of dislocations from the left boundary of the grain, with each subsequent $n$ dislocation starting in the slip plane $y_{n}=y_{n-1}+h$. In order to exclude the influence of dynamic effects [20-22], each subsequent dislocation was emitted only after equilibrium was established in a cluster consisting of previously emitted dislocations.

The equilibrium position of dislocations in the cluster formed after the generation of each new dislocation was found as follows. With a given initial time step, the coordinates of the cluster dislocations $x_{i}$ were determined at which the total stress acting on each of them turned out to be less than or equal to the threshold stress of dislocation start $\sigma^{\text {th }}$. Then this procedure was repeated with a smaller time step and new coordinates of dislocations $x_{i}^{\prime}$ were determined. The time step $\Delta t$ decreased until the coordinate changes $\Delta x_{i}=x_{i}-x_{i}^{\prime}$ of each $i^{\text {th }}$ cluster dislocation became of the order of magnitude of the Burgers vector of the dislocations $\left(\sim 2.5 \cdot 10^{-4} \mu \mathrm{m}\right)$. Such a calculation method allows one to determine with good accuracy the stable equilibrium position of the system under consideration.

\section{Results of computer simulation}

As the simulation results show, in the behavior of the band front as it passes through the disclination elastic field, general regularities are observed. For a given distance between the slip band and disclination $y_{0}$, the behavior of the cluster essentially depends on the distance $h$ between the slip planes of the dislocations. Fig. 2 shows the equilibrium configurations of dislocation cluster retarded by the wedge disclination of strength $\omega=0.04$ in the slip band located at a 
distance $y_{0}=0.5 \mu \mathrm{m}$ at $h=20 b$ (Fig. $2 \mathrm{a}$ ) and $h=50 b$ (Fig. $2 \mathrm{~b}$ ) and $\sigma^{\text {ext }}=0.01 D$.

For $h=20 b$ (Fig. 2 a), the behavior of the cluster is similar to the behavior of a dislocation pile-up, considered in [12]. It is noteworthy that the highest dislocation density does not arise in the cluster head, as in the classical dislocation pile-up inhibited near the barriers [23], but in its central part (Fig. $2 \mathrm{a}$ ). An increase in the distance $h$ between the slip planes of the dislocations leads to a "splitting" of the initial front of the band (Fig. 2 b). At large $h$, the accumulation of dislocations in the front of the band is rearranged into a configuration that is a dislocation wall broken from both sides. The equilibrium configuration of cluster dislocations at $h=75 b, \sigma^{\text {ext }}=0.01 D$, $y_{0}=0.5 \mu \mathrm{m}, \omega=0.04, N=15$ is shown in Fig. $3 \mathrm{~d}$.

In addition to the "quasistatic" method of forming an equilibrium cluster described above, the case was also considered closer to reality when the cluster was formed first at an obstacle (fixed head dislocation) in the absence of a disclination field, and then moved as a whole from the left boundary of the computational domain under the

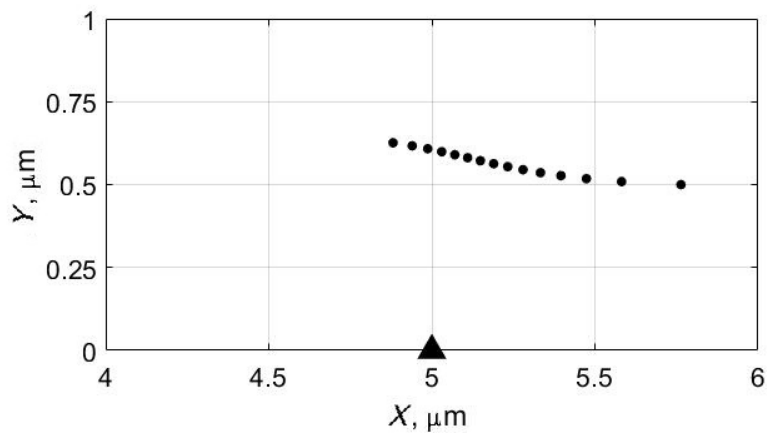

Fig. 2. Equilibrium configurations of dislocation in the front of the band at $h=20 b(\mathrm{a})$ and $h=50 b(\mathrm{~b}) .\left(\sigma^{\text {ext }}=0.01 D, y_{0}=0.5 \mu \mathrm{m}, \omega=0.04, N=15\right)$.

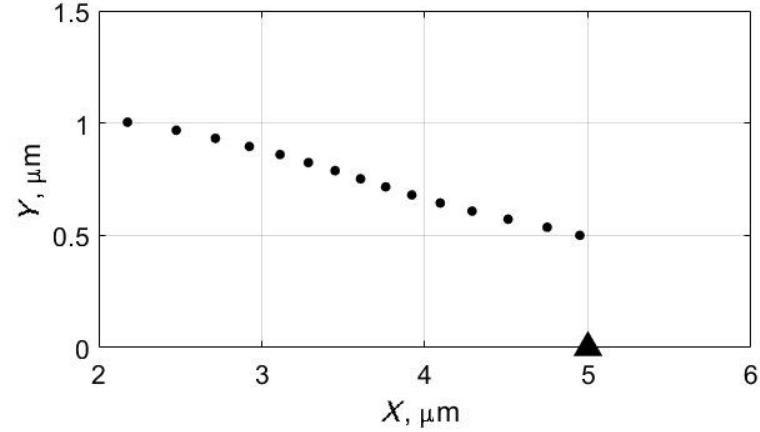

a

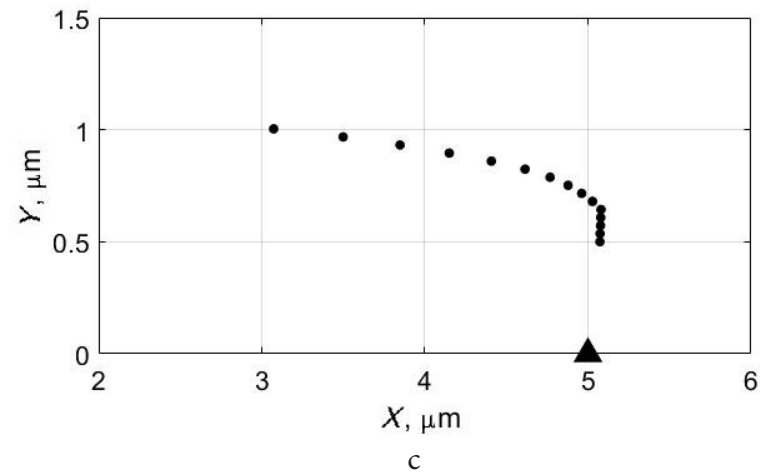

action of an external stress field towards the disclination. The analysis shows that when a certain minimum distance $h_{c}$ is exceeded and when external stresses are not too large, an equilibrium wall of dislocations broken from two sides also forms near the disclination. Fig. 3 shows the successive stages of transformation of the thus formed front of the band at $\sigma^{\text {ext }}=0.01 D, y_{0}=0.5 \mu \mathrm{m}, \omega=0.04, N=15$ for the dislocation mobility $M=1.5 \cdot 10^{6} \mathrm{D}^{-1} \mathrm{~s}^{-1}$. In this case, the final configuration of the wall turned out to be the same as that formed during the "quasistatic" method of its formation. The analysis shows that the minimum distance $h$, beyond which a dislocation wall broken from both sides is formed, depends on the disclination strength $\omega_{0}$ and the distance $y_{0}$ from the slip band to the disclination.

Fig. 4 shows the dependence of the misorientation of the forming wall $\omega=b / h$ normalized to the disclination power $\omega_{0}$. Note that at $y_{0}=0$, dislocation walls may be formed formed with the maximum possible misorientation $\omega^{\max }=\omega_{0}$.

With a gradual increase in the external stress $\sigma^{\text {ext }}>0.01 D$, the equilibrium cluster configurations (Fig. 2a,b, Fig. 3d)

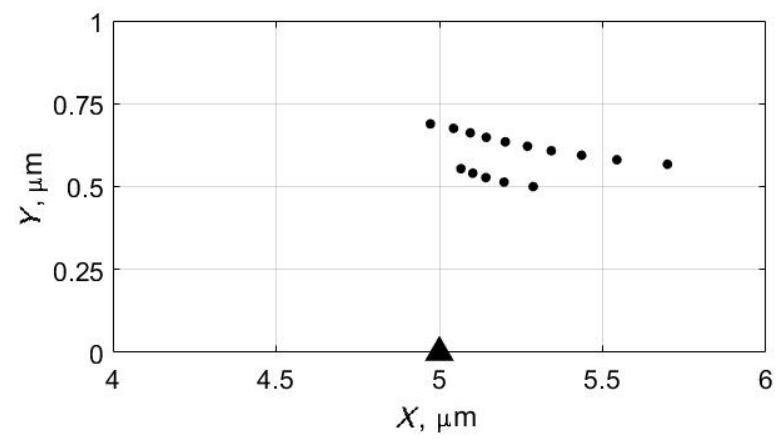

b

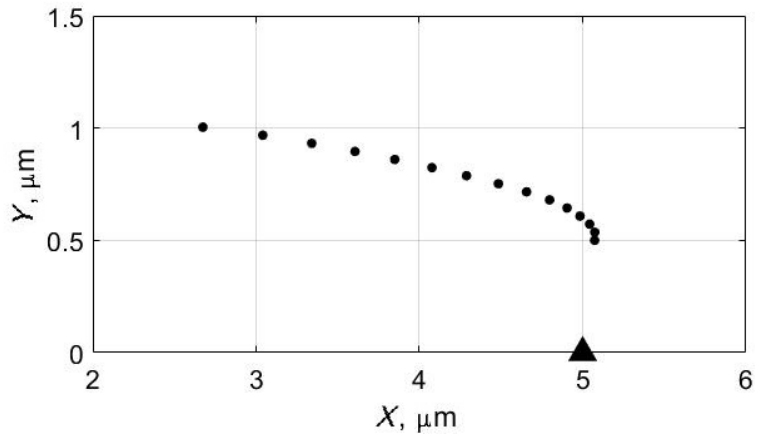

b

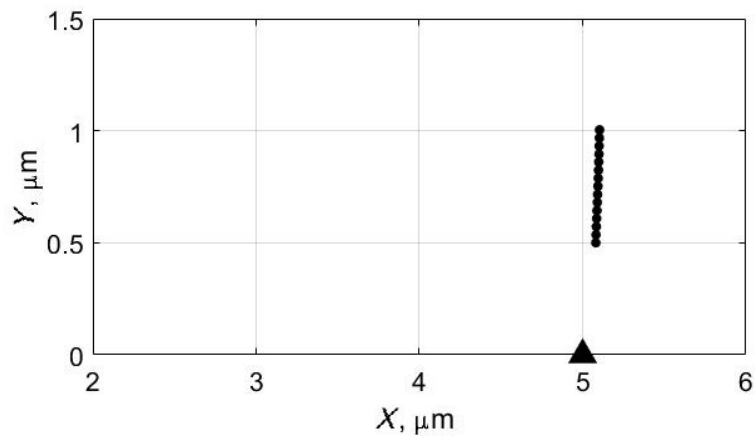

$\mathrm{d}$

Fig. 3. The successive stages of transformation of the slip band front into the broken dislocation wall at $h=75 b: t=0.1 \mathrm{~s}$ (a), $t=0.2 \mathrm{~s}$ (b), $t=0.3 \mathrm{~s}(\mathrm{c}), t=1.5 \mathrm{~s}(\mathrm{~d}) .\left(\sigma^{e x t}=0.01 D, y_{0}=0.5 \mu \mathrm{m}, \omega=0.04, N=15, M=1.5 \cdot 10^{6} D^{-1} \mathrm{~s}^{-1}\right)$. 


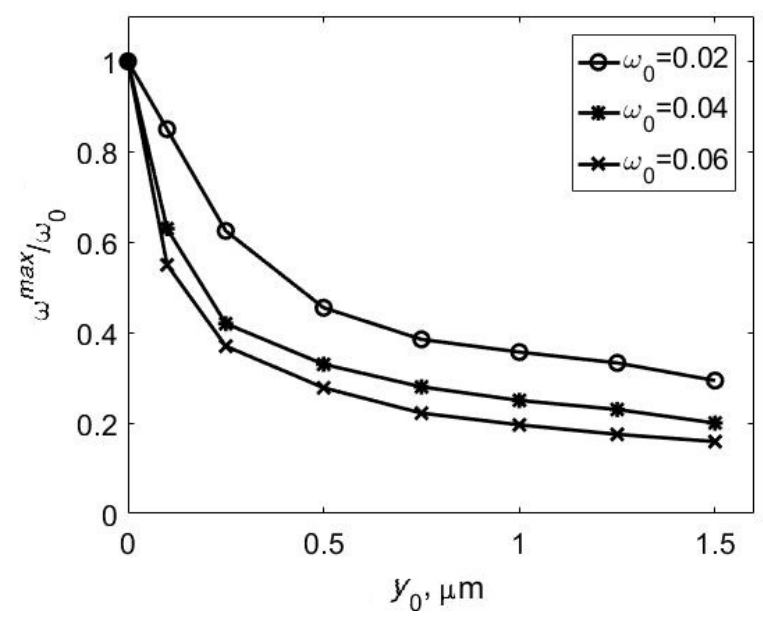

Fig. 4. Dependence of the maximal misorientation of the broken dislocation boundary $\omega^{\max }$, normalized by the value $\omega_{0}$, on the distance between the disclination and the slip band $y_{0}$.

formed at the parameters $h$ and $y_{0}$ considered above move to the right, while their equilibrium configuration retains as a whole its similarity. When the external stress reaches a value greater than a certain critical value $\left(\sigma^{\text {ext }} \geq \sigma_{c}\right)$, clusters lose their stability. So, for the case shown in Fig. 2 a, when $\sigma^{\text {ext }}>\sigma_{c}=0.011 D$, the head dislocation "breaks away" from the cluster with its subsequent movement and sink on the right side surface of the crystal. For the case of a "split" front of the band (Fig. $2 \mathrm{~b}$ ) with $\sigma^{\text {ext }} \geq 0.012 D$, such process occurs for the dislocation of the cluster that is most distant from the disclination. Finally, in the case when a broken wall of dislocations is formed (Fig. 3d), it overcomes the force barrier of disclination at $\sigma^{\text {ext }}>\sigma_{c}^{*}=0.02 D$ and moves to the right side surface of the crystal.

Note that, due to the cylindrical symmetry of the elastic disclination field, such walls can be formed with an arbitrary orientation of the slip band relative to the crystal lattice of the grain. Therefore, in the general case, a situation is possible when the front of the slip band passes through the force disclination barrier in the grain body, it is possible that not one dislocation wall broken from both sides appears, but systems of such broken dislocation boundaries with the same charge parallel to each other.

The critical stress of plastic shear in the case of wall formation reaches a maximum value and coincides with the stress for passage of a single dislocation. Thus, the behavior of

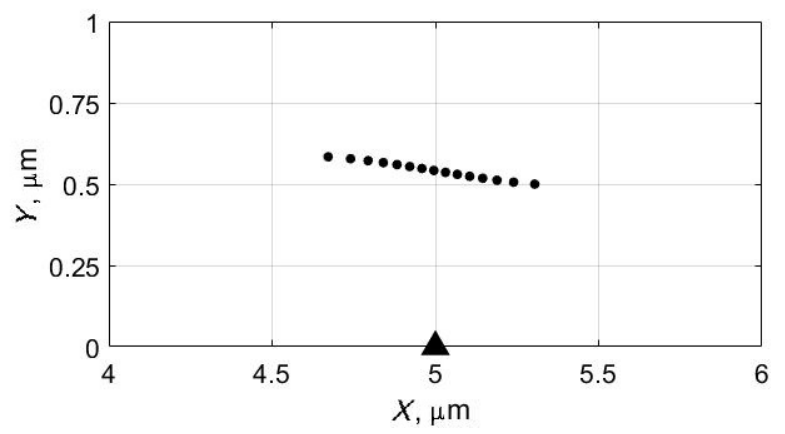

a the dislocation wall broken from both sides is similar to the behavior of a superdislocation with a Burgers vector $B=b N$ and, therefore, the stress necessary for its passage through the disclination force barrier does not depend on the distance between the slip band and the disclination.

Thus, the model predicts the formation of specific dislocation structures near the junction disclinations in the form of distributed clusters or dislocation walls broken from both sides. It should be noted that these structures do not disappear when the external load is removed, but relax to new equilibrium configurations, retaining as a whole their similarity. As an illustration of it, Fig. 5 shows the result of the transformation of the structures shown in Fig. 2, after removing the external load. Note that in the case of the formation of the wall, its configuration remains unchanged after removing the external load.

\section{Conclusions}

1. The interaction of the front of the slip band with the elastic field of the wedge disclination is considered. It is shown that in the process of passing of a plastic shear through the force barrier of the disclination, both the "splitting" of the front of the band and formation of the broken from both sides dislocation wall is possible. The conditions necessary for the formation of broken dislocation walls are determined. It was shown that the maximum possible misorientation of such dislocation boundaries depends on the disclination power and the distance between the slip band and the disclination.

2. Junction disclinations under conditions of the plastic flow localization in the form of slip bands can act as a "generators" of systems of broken from two sides dislocation boundaries parallel to one another.

Acknowledgements. The work was performed in the framework of the state assignment for conducting fundamental scientific research for 2013-2020 by the IAP RAS, task No. 0035-2014-0401.

\section{References}

1. V.V. Rybin. Large plastic deformations and fracture of metals. Moscow, Metallurgiya (1986) 223 p. (in Russian)

2. V.V. Rybin, A. A. Zisman, N. Yu. Zolotorevsky. Acta Met. Mater. 41 (7), 2211 (1993). $\underline{\text { Crossref }}$

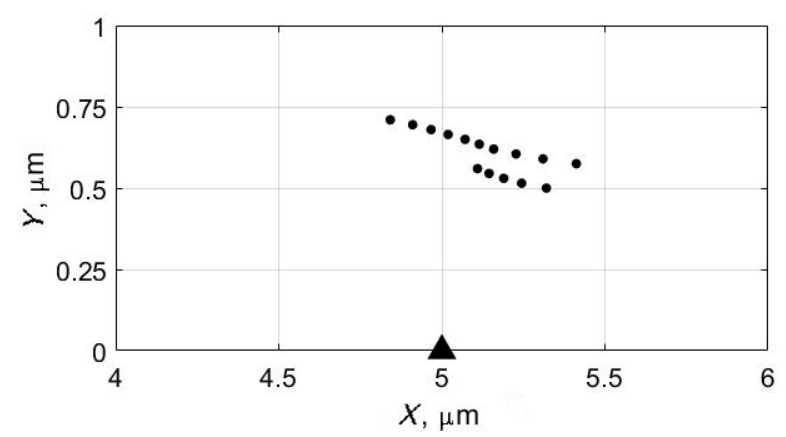

b

Fig. 5. Equilibrium configurations of dislocations after unloading at $h=20 b$ (a) and $h=50 b$ (b). 
3. V.V. Rybin, A.A. Zisman, N. Yu. Zolotorevsky. Phys. Solid State. 27 (1), 181 (1985) (in Russian)

4. V.N. Perevezentsev, G. F. Sarafanov, J. V. Svirina. Mater. Phys. and Mech. 21 (1), 78 (2014).

5. V.N. Perevezentsev, G. F. Sarafanov. Mater. Sci. Eng (a). 503 (1-2), 137 (2009). Crossref

6. V.N. Perevezentsev, G. F. Sarafanov. Tech. Phys. Lett. 31 (11), 936 (2005). Crossref

7. G. F. Sarafanov, V. N. Perevezentsev. Letters on Materials. 1 (1), 19 (2011). (in Russian) Crossref

8. G.A. Salishchev, S. Yu. Mironov, S.V. Zherebtsov, A. N. Belyakov. Mater. Phys. and Mech. 25, 42 (2016). (in Russian)

9. N.Yu. Zolotorevsky, V.V. Rybin, A.N. Matvienko, E. A. Ushanova, S. N. Sergeev. Letters on Materials. 8 (3), 305 (2018). (in Russian) Crossref

10. M. Seefeldt. Rev. Adv. Mater.Sci. 2, 44 (2001).

11. V. V. Rybin, V. N. Perevezentsev, J. V. Svirina. Mater. Phys. and Mech. 32 (3), 237 (2017).

12. V. V. Rybin, V.N. Perevezentsev, J. V. Svirina. Tech. Phys. The Russian Journal of Applied Physics. 63 (7), 974 (2018). Crossref

13. E. Van der Giessen, A. Needleman. Modelling Simul. Mater. Sci. Eng. 3, 689 (1995). $\underline{\text { Crossref }}$
14. M.S. Wu. International Journal of Plasticity. 100, 142 (2018). Crossref

15. V.N. Perevezentsev, V.V. Rybin, V.N. Chuvildeev. Acta Metallurgica et Materialia. 40 (5), 887 (1992). Crossref

16. V.V. Rybin, I. M. Zhukovsky. Phys. Solid State. 20 (6), 1829 (1978). (in Russian)

17. T. Wang, J. Luo, Z. Xiao, J. Chen. Eur. J. Mech. - A/Solids. 28, 688 (2009). Crossref

18. G. F. Sarafanov, V. N. Perevezentsev. Russian Metallurgy (Metally). 10, 889 (2016). $\underline{\text { Crossref }}$

19. A.E. Romanov, V.I. Vladimirov. Disclinations in crystalline solids. In: Dislocations in solids (ed. by F. R. N. Nabarro). Vol. 9. Amsterdam, Elsevier Science Publishers (1992) p. 191-402.

20. E. A. Rzhavtsev, M. Yu. Gutkin. Scripta Materialia. 100, 102 (2015). Crossref

21. S. V. Dmitriev, A.I. Pshenichnyuk, A. M. Iskandarov, A. A. Nazarova. Model. and Simul. in Mater. Sci. and Eng. 18 (2), 025012 (2010). $\underline{\text { Crossref }}$

22. D. V. Bachurin, R.T. Murzaev, Yu.A. Baimova, A. A. Samigullina, K. A. Krylova. Letters on materials. 6 (3), 183 (2016). (in Russian) Crossref

23. J.P. Hirth, J. Lothe. Theory of dislocations. New York, Wiley (1982) 839 p. 